\title{
Approximate Volumetric Reconstruction from Projected Images
}

\author{
Gabor Fichtinger ${ }^{1}$, Sheng Xu ${ }^{1}$, Attila Tanacs ${ }^{1}$, Kieran Murphy ${ }^{2}$, Lee Myers ${ }^{3}$, \\ Jeffery Williams ${ }^{4}$, \\ ${ }^{1}$ Center for Computer Integrated Surgical Systems and Technologies, \\ Johns Hopkins University, Baltimore, MD, 21218 \\ 3400 N. Charles St, New Engineering Bldg B26 \\ contact: gabor@cs.jhu.edu \\ ${ }^{2}$ Department of Radiology, Johns Hopkins University Hospital, Baltimore, MD \\ ${ }^{3}$ Department of Radiation Oncology, Johns Hopkins University Hospital, Baltimore, MD \\ ${ }^{4}$ Department of Neurosurgery, Johns Hopkins University Hospital, Baltimore, MD
}

\begin{abstract}
A significant problem in planning of volumetrically prescribed localized treatments is the mathematical impossibility to determine the exact three dimensional shape and volume of a target object from its projected images. Reconstruction accuracy also varies with viewing angle, depending on the convexity and aspect ratios of the target object. In response to this problem, we are developing a robust and efficient technique for approximate volumetric reconstruction, which (A) uses no prior information of the shape and volume of the target, (B) does not require exact silhouettes, (C) accepts arbitrary number of images, (D) produces solid object and measure of its volume, (E) provides confidence measure of the reconstruction and drawing of silhouettes, $(F)$ is robust, fast and easy to implement. Preliminary tests suggest that fairly convex objects can be reconstructed from four views, and typically six views with table rotation allow us to reconstruct fine details as small as $1 \mathrm{~mm}$. The method is applicable for any X-ray guided volumetric treatment. Pilot applications will be planning of radiosurgery of arterioveneous malformations (AVMs) and radiofrequency ablation of soft tissue lesions.
\end{abstract}

\section{Introduction}

Contrast-enhanced angiography is the primary imaging modality in AVM radiosurgery and it has assumed significant role in minimally invasive local therapies. A fundamental obstacle in planning such therapies is the lack of reliable volumetric appreciation of the target. The problem has not been researched during the last half decade since fusion of angiography to CT/MRI had become available for AVM radiosurgery planning. At the same time, even state-of-the-art radiosurgery systems, like BrainLAB or Leibinger, merely back-project the silhouettes to reconstructed $\mathrm{CT} / \mathrm{MRI}$ planes, without giving volumetric appreciation of the target. In general, it is impossible to reconstruct an object exactly in 3D from its projective images. Worse yet, in angiography images the target does not present well defined boundaries and the doctor must depend on subjective clinical judgment when outlining the target 
object (Figure 1.) Current methods fail to address the fact that contours, as subjectively drawn by the physician, do not necessarily correspond to a three-dimensional object. Several prior volumetric reconstruction methods exist that also place limitations on the number of projections and the rotational freedom of the X-ray source [1,3]. In 1996, during the peak of interest in mono-modal angiographic planning of AVM radiosurgery, Yeung et al. [2] proposed a method based on pure back-projection from contours. The paper, to-date perhaps the most

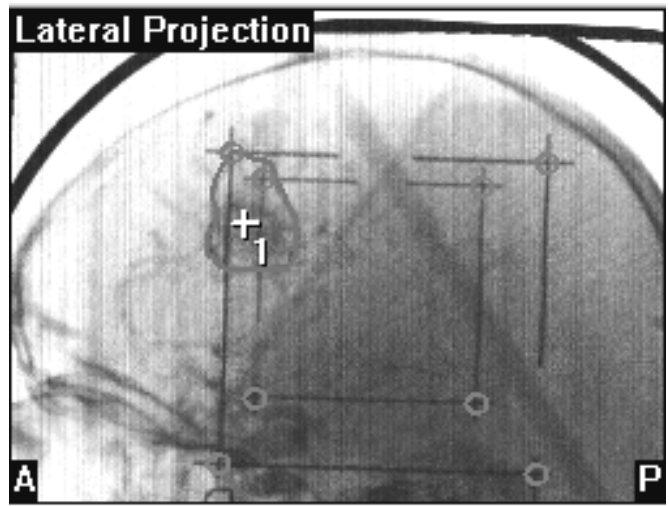

Figure 1: Stereotactic angiogram of an AVM for radiosurgery planning. The target is contoured by the physician around the + marker. general and robust method, underestimated the problem that back-projection alone produces only a cloud of disjoint voxels that has to be solidified later, in order to receive a solid $3 \mathrm{D}$ object. The paper also did not address directional robustness and consistency of contours. Parallel to medical applications, a family of shape recovery methods have also emerged in the fields of pattern recognition and computer vision $[3,4,5,6]$. These algorithms, besides being rather complex and difficult to implement, deal with solid physical objects with well defined boundaries, therefore, are not suitable for our purpose.

\section{Methods}

We propose a combination of forward- and back-projections for reconstruction that begins after the surgeon draws the silhouettes of the target in each 2D image. From back-projections of the silhouettes, we determine a closest fitting regular shape that is guaranteed to cover the object in 3D. Then using primarily forward projection, all excess parts are carved off the encompassing shape, till we receive the reconstructed object. In the most conservatively approach, the object must fit inside all silhouettes. Multiple methods were developed to carry out this phase. A fast algorithm takes advantage of occasional convexity of silhouettes, while a voxel-by-voxel method works for all shapes of silhouettes. Finally, the obtained object is projected forward on to each image plane, where the shadow of the reconstructed object is compared to the silhouettes drawn by the surgeon, so that confidence and consistency of silhouette lines could be calculated and visually interpreted. Objects with large aspect ratio inevitably have preferential directions allowing significantly more accurate reconstruction than other projection angles. Part of our robustness analysis is quantitative prediction for the inaccuracy of reconstruction caused by sub-optimal projection angles. 


\section{Current Status}

Currently we are experimenting with synthetic data generated by forward-projection of various objects onto the planes of a hypothetic imager. Objects that are convex in the center of gravity and that have no large aspect ratio can be reconstructed with high accuracy from three or four images. According to expectations, objects with large aspect ratio were reconstructed significantly more accurately from perpendicular directions than from parallel directions, with respect to their long axis. Figure 2 shows a highly complex synthesized tree-branch phantom, similar to the one reported by Yeung [2]. When the object was projected in preferential directions, the relative error was $4.3 \%, 3.6 \%$, and $0.8 \%$ from 3,5 , and 7 shots, respectively. When the same object was projected in random directions, the mean relative

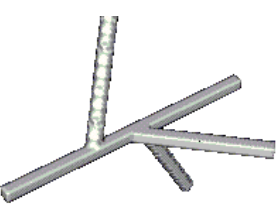

Figure 2: Synthetic tree-branch phantom error was $11.3 \%$ in 20 random experiments, from seven projections in each. Based on visual inspection, our results appear to be at least as accurate as Yeung's who did not report numbers. Imperfect silhouettes with known measures of inconsistency have been also synthesized, numerical evaluation of these is in progress. While still more phantom and synthetic studies will be carried out, clinical robustness will also be examined in post-operative analysis of fluoroscopic images of liver lesions and intracranial AVMs.

\section{Acknowledgements}

The project is supported by the National Science Foundation under the Engineering Research Center grant \#EEC9731478.

\section{References}

[1] Foroni, R.; Gerosa, M. Shape recovery and volume calculation from biplane of atreriovenous malformations. Rad. Onc. Biol. Phys., Vol. 35, No.3, pp. 565-577

[2] Yeung D; Chen N; Ferguson RD; Lee LI; Kun LE Three-dimensional reconstruction of arteriovenous malformations from multiple stereotactic angiograms. Med Phys 1996 Oct;23(10):1797-1804

[3] Chang, S.K.; Wang, Y. R. Three dimensional object reconstruction from orthogonal projections. Patt. Recongnit. 7:167-176; 1975

[4] Xu, G.; Tsuji, S. Recovering surface shape from boundary. Int. Conf. Art. Intel. 2:731$733 ; 1987$

[5] Ulupinar, F.; Nevatia, R. Shape from contours: SHGCs. In: Proc. IEEE Int. Conf. Computer Vision 582; 1990

[6] Ulupinar, F.; Nevatia, R. Inferring shape from contours for curved surfaces. Atlantic City: Proceeding International Conference Pattern Recognition. 1990: 147-154. 